\title{
Entre expérimentation réglée et expérience vécue
}

Les dimensions subjectives de l'activité de l'ergonome en intervention Modeling and teaching the subjective dimensions of ergonomic practice

\section{François Daniellou}

\section{(2) OpenEdition}

\section{Journals}

Édition électronique

URL : http://journals.openedition.org/activites/1835

DOI : 10.4000/activites. 1835

ISSN : 1765-2723

Éditeur

ARPACT - Association Recherches et Pratiques sur les ACTivités

Référence électronique

François Daniellou, «Entre expérimentation réglée et expérience vécue ", Activités [En ligne], 3-1 | avril 2006, mis en ligne le 01 avril 2006, consulté le 20 avril 2019. URL : http://journals.openedition.org/ activites/1835; DOI : 10.4000/activites.1835

\section{cc) (†) $\ominus$}

Activités est mis à disposition selon les termes de la licence Creative Commons Attribution - Pas d'Utilisation Commerciale - Pas de Modification 4.0 International. 


\title{
Entre expérimentation réglée et expérience vécue : Les dimensions subjectives de l'activité de l'ergonome en intervention
}

\author{
François Daniellou \\ Laboratoire d'Ergonomie des Systèmes Complexes \\ Université Victor Segalen Bordeaux 2 \\ Case 55, 146 rue Léo-Saignat, 33076 Bordeaux Cedex \\ Francois.Daniellou@ergo.u-bordeaux2.fr
}

\begin{abstract}
Modeling and teaching the subjective dimensions of ergonomic practice. The models of operators' activity, which are produced in ergonomics, will increasingly include a reference to its subjective dimensions. Ergonomist's activity models have not followed the same development. To foster the construction of professional practices, it appears necessary to model and to teach the subjective dimensions of the ergonomist's activity.
\end{abstract}

KEYWORDS

Activity analysis, ergonomic practice, intervention, subjectivity.

Longtemps, l'ergonome s'est intéressé au travail des autres. D'abord à celui d'opérateurs «de base », qu'ils soient ouvriers, employés, contrôleurs aériens ou de processus continus. Plus récemment, l'activité des concepteurs, des organisateurs, des cadres a fait l'objet de son attention. Mais l'ergonome lui-même ou elle-même est aussi un opérateur ou une opératrice, dont l'activité, notamment d'intervention, peut être analysée.

Nous rappellerons d'abord quelques aspects et enjeux du développement de la recherche sur l'intervention ergonomique. Puis nous discuterons dans quelle mesure les modèles de l'activité appliqués à l'ergonome ont suivi le développement des modèles plus généraux de l'activité mis en œuvre en ergonomie : le modèle du sujet-ergonome n'est-il pas devenu plus pauvre que le modèle du sujetopérateur couramment utilisé en ergonomie?

Nous argumenterons pour une meilleure prise en compte des dimensions subjectives de l'activité de l'ergonome dans les modèles qui essaient de rendre compte de cette dernière, notamment aux fins d'enseignement. Cette nécessité est certainement plus ou moins forte suivant les classes de situations dans lesquelles l'ergonome intervient. Nous limiterons notre propos aux interventions ergonomiques portant sur la conception ou la transformation de situations de travail.

\section{1.- Modéliser l'activité de l'ergonome : un champ de la recherche en ergonomie}

L'intérêt de modéliser l'activité transformatrice de l'ergonome semble maintenant admis (Pinsky, 1992 ; Daniellou, 1992, 1996, 1999 ; Falzon, 1997 ; Lamonde, 2000). Prendre l'activité des ergonomes comme objet de formalisation répond à trois enjeux : 
- Il s'agit d'abord de mieux élucider les mécanismes par lesquels les ergonomes contribuent à la transformation des situations de travail (Daniellou, 1992). L'hypothèse naguère implicite (par exemple dans Guérin, Laville, Daniellou, Duraffourg, \& Kerguelen, 1991, 1997) était que leurs analyses des situations de travail, et leurs connaissances générales sur l'homme au travail, permettaient de déboucher sur des « recommandations » qui seraient mises en œuvre de façon autonome par les acteurs de l'entreprise concernée. Elle paraît aujourd'hui bien faible pour rendre compte de leurs actions, de leurs succès et de leurs échecs.

- Il s'agit ensuite de mettre à la disposition des professionnels des formes de conceptualisation qui favorisent leur réflexion dans l'action, les discussions entre eux, la gestion des crises, et plus généralement l'élaboration des règles de métier (Martin, \& Baradat, 2003).

- Il s'agit enfin de favoriser l'enseignement et la transmission du métier (Lamonde, 2000).

Nous avons décrit ailleurs (Daniellou, 1999 ; Petit, Querelle, \& Daniellou, à paraître), les différentes méthodologies qui pouvaient être utilisées pour analyser et modéliser l'activité de l'ergonome, selon que l'observateur-analyste est ou non membre de l'équipe d'intervention. Lamonde (2000) insiste sur les différents types de verbalisation qui peuvent être mis en œuvre, et sur les difficultés qu'ils comportent.

L'objet de cet article n'est donc pas d'argumenter à nouveau l'intérêt d'une analyse de l'activité de l'ergonome, notamment en intervention. Il s'agit de revenir sur les modèles mêmes de l'activité qui sont mis en œuvre pour décrire celle de l'ergonome, et d'évaluer dans quelle mesure ceux-ci ont suivi l'enrichissement des modèles du sujet et de l'activité mobilisés par ailleurs en ergonomie.

\section{2.- Une évolution des modèles de l'activité}

Dans un ouvrage récent, Rabardel et Pastré (2005) ont rassemblé un ensemble de contributions relatives aux « Modèles du sujet pour la conception». On peut trouver dans le titre un lointain écho à la célèbre question de Wisner (1971) : «À quel homme le travail doit-il être adapté ? ». Entre les deux textes, dont les titres reflètent sans doute une même préoccupation, un chemin considérable a été parcouru, comportant bien sûr autant de difficultés nouvelles que de voies pour l'action ergonomique.

Le texte de Wisner avait un objectif limité : rappeler la diversité et la variabilité des caractéristiques physiques des êtres humains, face au concept taylorien d' « homme moyen ». Il n'est en rien représentatif du modèle de l'être humain dont était porteur son auteur, dont on connaît la culture médicale, historique et sociale. Mais il souligne le type de débats qu'il était nécessaire de faire avancer voilà trente-cinq ans.

Aujourd'hui, le concept que Rabardel et Pastré jugent nécessaire de mettre en avant pour éclairer l'intervention en conception est celui de «sujet». Le projet est explicite : "Les travaux sur les dimensions cognitives de l'activité sont si développés qu'ils finissent par donner une représentation distordue des sujets humains. Il faut, au plan de la recherche, développer des connaissances mieux équilibrées et plus représentatives de l'humain. ${ }^{1}$ (Rabardel, 2005, p. 13). Les auteurs dressent le portrait d'un « sujet capable», en développement, qui mobilise un ensemble de ressources internesexternes, mais qui ne se limite pas aux ressources dont il dispose : «Mais survient une crise, une rupture, et l'on peut voir alors que le sujet ne peut pas se confondre avec l'organisation de son activité : il est irréductible et insubstituable par rapport à elle. » (Rabardel et Pastré, op. cit., p. 4).

1. L'alerte par rapport à l'accent exclusif mis sur les dimensions cognitives n'est évidemment pas nouvelle. J. Bruner, l'un des pères de la « révolution cognitive » y a par exemple contribué lui-même, en publiant dès 1990 « ...Car la culture donne forme à l'esprit » (Bruner, 1990). 
Pastré (2005a, pp. 231 sqq) fait travailler la notion de "genèse » jusqu'à parler de "genèse identitaire » pour évoquer la reconstruction du sujet quand celui-ci « sous le coup des circonstances, n'arrive plus à se reconnaitre dans le genre ou le monde dans lesquels il avait l'habitude d'évoluer. » (p. 259).

Cette prise de position, la dernière en date, s'inscrit évidemment dans une longue série d'interpellations de l'ergonomie sur les modèles des êtres humains qu'elle met en œuvre quand elle analyse leur activité. Nous avons essayé de retracer une partie de l'histoire de ces alertes dans (Daniellou, 2005). La psychodynamique (Dejours, 1981), l'ergologie (Schwartz, 2000), la clinique de l'activité (Clot, 1999), les travaux de Curie (2000), ont par exemple, avec des arrière-plans théoriques différents, tous contribué à faire évoluer les regards sur l'activité humaine au sein de la communauté « de l'ergonomie de l'activité ». Certes, ces différentes approches portent des regards distincts sur les potentialités vitales du sujet et sur ce qui les bride, ou sur les liens entre connaissance de l'être humain au travail et action transformatrice. Mais nous ne chercherons pas ici à décrire les différences souvent importantes entre elles. Nous voudrions plutôt retenir que les modèles généraux de l'activité humaine au travail aujourd'hui mobilisés en ergonomie intègrent fréquemment les dimensions suivantes.

1) L'activité ne peut se limiter à « ce qui est mis en œuvre pour réaliser les tâches ». L'activité est une tranche de vie, dont les déterminants i) ne sont pas tous liés au travail, ii) ne sont pas tous présents dans la fenêtre géographique et temporelle d'observation. L'activité trouve ses mobiles et certains de ses buts dans l'ensemble de l'histoire de la personne, dans ses dimensions professionnelles et non-professionnelles. Il est impossible, par exemple, de comprendre les compromis coûteux qu'un opérateur posté fait vis-à-vis des horaires de travail si l'on n'a pas entendu « qu'il était prêt à faire ça jusqu'à ce que sa fille ait son bac ». La multiplicité des déterminants n'implique pas que l'ergonome doive en explorer l'ensemble à chaque fois, mais plutôt qu'il/elle ne peut définir a priori le périmètre de ce qui sera essentiel pour comprendre une activité de travail.

2) L'ensemble de la biographie de la personne, avec ses aspects familiaux, sociaux, culturels, a contribué à structurer chez elle un ensemble de valeurs qui l'amène à ne pas valoriser de façon équivalente toutes les composantes et tous les produits de son activité.

Ces valeurs portées par la personne entrent en résonance et en confrontation avec des normes et des valeurs présentes dans la situation de travail. Ces autres «normes antécédentes » (Schwartz, 2000, p. 594) proviennent pour partie de la loi, du règlement intérieur de l'entreprise, des procédures, des consignes, des matières travaillées et outils mis à disposition. Pour partie aussi, elles ont été forgées par l'histoire des collectifs de travail, des « entités collectives relativement pertinentes » par rapport à l'activité du sujet concerné (ibid., p. 604). Pour partie enfin, elles proviennent des clients (ou usagers, patients, élèves, ...).

3) Ces normes et valeurs ne sont pas spontanément compatibles, mais au contraire fréquemment sources de contradictions. Leur traitement ne relève pas d'une «optimisation», car elles sont de natures hétérogènes, et il n'existe pas d'équivalent général auquel elles pourraient être ramenées. Par exemple, Dejours (1995 p. 79) reprend les termes d'Habermas pour évoquer les rationalités de l'agir (efficacité dans le monde physique, justesse dans le monde social, authenticité dans le monde subjectif), et les délibérations auxquelles elles donnent lieu. Tous les objectifs, qui relèvent de différentes rationalités, ne peuvent souvent pas être atteints à la fois, et Clot (1999) a souligné que ce qui était coûteux dans le travail n'était pas seulement ce qu'on fait, mais aussi ce que l'on cherche à faire sans y parvenir.

4) Chacun est ainsi, dans le travail, confronté à un « débat de normes », entre « usage de soi par soi » et « usage de soi par les autres » (Schwartz, 2000), auquel il prend part « activement », c'est-à-dire en cherchant par son activité à en influencer l'issue, à être pour quelque chose dans les règles qui prévaudront dans les faits, à « renormaliser le milieu » (Canguilhem, 1966). 
5) Ces délibérations sur des valeurs contradictoires sont constitutives de l'humain comme « sujet» (Schwartz parle du « corps-soi », Bruner du « self»). Les formulations sont différentes... Curie (2000 p. 19) indique qu'il a cherché à « analyser les processus de personnalisation, c'est-à-dire les activités d'un individu se comportant en sujet par ses délibérations sur des valeurs contradictoires, des procédures antagonistes, des projets incompatibles ou contradictoires, et restructurant au terme provisoire de ces délibérations les systèmes internes, cognitifs, conatifs et axiologiques organisateurs de ses conduites dans les situations de conflit». Pastré (2005a, p. 259) écrit : «Les organisateurs de l'activité constituent des ressources d'ensemble à la disposition du sujet, mais ne se confondent pas avec lui. Le sujet dispose de ces ressources ; il peut même leur déléguer l'orientation de son action; il peut augmenter ses ressources ou les réorganiser quand le besoin s'en fait sentir. Mais il existe des moments, et les moments de rupture fondatrice sont exemplaires sur ce point, où il faut bien admettre qu'il n'y aurait pas de sujet sans transcendance par rapport aux organisateurs de l'activité ». Schwartz évoque le « corps-soi », « ce corps, ce même corps, qui tente de faire face à toutes les situations de la vie, ce "soi" de l'usage de soi, à l'épreuve de toutes les circulations entre "travail" et "non-travail", ce corps qui lie le synchronique au diachronique, est tout ensemble le corps biologique, le corps "biographique" qui porte les stigmates de sa tentative d'inscription dans l'être social, le corps parlant et signifiant, le corps culturel et historique » (2000 p. 609).

Toutes les questions adressées à l'ergonome ne nécessitent certes pas qu'il/elle mobilise une modélisation de l'humain au travail où les dimensions subjectives soient mises en avant. Dans la mesure où les modèles de l'activité produits par l'ergonome ne visent pas à décrire toutes les dimensions de l'activité, mais à servir de base à des transformations pertinentes, il est clair que, dans bien des cas, il peut améliorer significativement un environnement de travail avec des modèles plus réduits. Mais l'ergonome ne peut jamais présager du niveau de modélisation de l'activité humaine qu'il sera nécessaire de mettre en œuvre pour comprendre ce qui se joue dans une situation donnée, et transformer celle-ci. Si des travailleurs s'exposent plus aux toxiques qu'ils ne pourraient le faire en adoptant une autre stratégie, c'est peut-être parce que, ce faisant, ils parviennent à augmenter leur salaire et donc à mieux financer les études de leurs enfants ; c'est peut-être parce que l'histoire du collectif valorise les prises de risque comme épreuve d'intégration, ou peut-être encore... Les actions transformatrices ne seront sans doute pas les mêmes dans l'un ou l'autre cas.

Les modèles classiquement mis en œuvre en ergonomie combinent dans des proportions variables une composante biologique (l'humain comme système de traitement de l'énergie), et une composante cognitive (l'humain comme système de traitement de l'information). Ce cadre « traitement de l'énergie/traitement de l'information » apparaît clairement comme trop étroit chaque fois par exemple que l'on analyse des activités de service, où l'on voit opérateurs et opératrices fréquemment confrontés à des débats de normes, entre ce que l'entreprise souhaite proposer comme service et ce qu'ils perçoivent comme la réponse juste à la situation du client (APST, 1992 ; Petit, 2005). Ce modèle est aussi insuffisant pour comprendre les difficultés des cadres, qui ont à faire respecter des règles dont ils ne sont pas les auteurs. Beaucoup d'autres situations de travail nécessitent une prise en compte des dimensions culturelles, axiologiques et subjectives de l'activité, notamment toutes les situations où l'objet du travail est des personnes.

Il nous semble qu'il en va ainsi de l'activité de l'ergonome. Le lecteur qui relirait les cinq points cidessus en les appliquant, non à une infirmière ou à un guichetier, mais à un-e ergonome pourrait-il considérer qu'ils perdent alors toute pertinence ? Il nous semble plutôt que l'enrichissement des modèles de l'activité appelle une évolution des modèles de l'intervention ergonomique qui servent de base, notamment, à l'enseignement de la pratique professionnelle. 


\section{3.- Les modèles de l'intervention sont-ils des reflets de, ou des instruments pour l'activité de l'ergonome?}

De nombreux travaux, nous l'avons dit, essaient de formaliser l'activité de l'ergonome dans l'intervention. Ils proposent des conceptualisations extrêmement utiles, dont nous allons chercher à préciser le statut, et les limites.

\section{1.- Statut des modélisations de l'intervention}

L'une des modélisations connues de l'intervention est celle qu'a faite Wisner, de façon synthétique dans « le diagnostic en ergonomie » (1972 [1995], pp. 96 sqq), beaucoup plus en détail dans son cours (1979). Ce modèle a ensuite été repris et explicité dans (Guérin et al., 1991). Il décrit un chemin de l'analyse de la demande au diagnostic, en passant par l'analyse ergonomique du travail dans des situations convenablement choisies.

Plus tard, des modélisations ont été proposées pour l'intervention dans des projets industriels (Daniellou, 1985 ; Daniellou, \& Garrigou, 1992), dans des projets architecturaux (Martin, 2000), dans des projets informatiques.

Lamonde (2000), dans le premier chapitre de son ouvrage, s'interroge : « les manuels de référence et les formations pour praticiens parlent-ils vraiment de la pratique professionnelle ?» et montre sans difficulté que les praticiens n'appliquent pas les démarches qui sont présentées dans les manuels. Mais l'écart entre les formalisations publiées et/ou enseignées, et les pratiques observées peut être interprété de différentes manières.

Une première interprétation - il semble que ce soit celle de l'auteure citée - consiste à considérer que ces modèles livresques auraient le statut d'une prescription de l'action des intervenants - et il est probable qu'il existe des lieux où ils sont enseignés sous cet angle ${ }^{2}$. Rien d'étonnant pour un ergonome, dès lors, que la prescription ne puisse pas être suivie à l'identique, et que des régulations soient mises en œuvre par les opérateurs(-ergonomes) qui ont la charge de la réaliser. Le raisonnement se déploie alors assez classiquement : si la prescription est à ce point éloignée de la réalité, c'est que les prescripteurs (en l'occurrence enseignants) n'ont pas une assez bonne connaissance du travail réel des intervenants.

Nous préférons considérer que les modèles enseignés servent à favoriser le développement de schèmes opératoires grâce à une conceptualisation, au sens de Vergnaud (1996). «Un schème n'est nullement un stéréotype mais une manière de régler son action en fonction des caractéristiques particulières de la situation ici et maintenant » (p. 281). «Ce qui est invariant c'est l'organisation de la conduite et non la conduite elle-même. [...] Un même schème peut engendrer des conduites relativement différentes en fonction des situations singulières auxquelles il est amené à s'adresser. Le décours temporel de la conduite (choix des actions, des prises d'information, des contrôles) peut ainsi suivre des trajectoires très différentes selon les valeurs prises par les variables de situation. » (pp. 283-284). Mais la constitution des schèmes à partir « de rien» est longue, et coûteuse. « La formation des connaissances opératoires consiste à la fois dans des gestes et pratiques difficiles à acquérir et dans des conceptualisations subtiles » (pp 284-285). L'un des enjeu des recherches en didactique professionnelle est de contribuer à une conceptualisation et de construire des mises en situation pédagogiques qui permettent d'accélérer et d'assurer cette construction (Pastré, 2005b).

Le rôle des enseignants-modélisateurs de l'activité ergonomique nous semble être typiquement celui-là : participer à l'élaboration de conceptualisations (de l'activité d'intervention) qui favorisent

2. Et beaucoup d'autres où, quelles que soient les précautions prises par les enseignants, des étudiants attribuent à ces modélisations un statut normatif. 
le développement de schèmes professionnels adaptables à une diversité de situations. En matière de conduite de projet industriel, par exemple, aucun projet ne se déroule du début à la fin suivant le schéma théorique qui en est fait, du fait des rebondissements inévitables (Jackson, 1998). Pour autant, les invariants « mise en place des structures de conduite de projet », « analyse de sites de référence », « recensement des situations d'action caractéristiques », « simulations », se retrouvent dans un très grand nombre de récits d'interventions, par ailleurs très différentes entre elles ${ }^{3}$.

Le rapport de l'ergonome-apprenant aux modèles d'intervention qui lui sont proposés ne nous semble donc pas être principalement un rapport de soumission à une prescription. Il nous semble qu'on peut mieux le décrire comme une « genèse instrumentale » (Rabardel, 1995), la modélisation ayant le statut d'un artefact, qui va faire l'objet du développement de schèmes chez l'apprenant. Mais l'artefact lui-même va être « mis à sa main » par l'intervenant, qui va le modifier pour mieux s'en servir.

Cette genèse instrumentale a lieu d'abord dans l'activité de chaque enseignant. Le célèbre schéma de Leplat et Cuny (1977 p. 55), devenu ensuite le «schéma à cinq carrés », est un instrument pour l'enseignant, c'est-à-dire que son utilisation fait l'objet du développement de schèmes personnels (construction progressive, commentaires). Il serait extrêmement intéressant de comparer les usages qui en sont faits dans différents lieux de formation.

Cette genèse instrumentale a lieu aussi chez chaque ergonome en formation, qui s'approprie les formalisations qui lui sont proposées dans les situations, par exemple de stages, qu'il/elle est amené-e à vivre. Elle se poursuit ainsi tout au long de sa carrière professionnelle. « Ce sont les obstacles, les discordances, les conflits objectifs, subjectifs ou intersubjectifs rencontrés dans l'activité qui génèrent une intensité plus ou moins forte de tension, et qui invitent le sujet à mobiliser et développer les invariants. » (Béguin, \& Clot, 2004, p. 45).

Mais - ce n'est pas le cas de toutes professions - la communauté ergonomique « de l'activité »s'est aussi donné les moyens d'une genèse instrumentale partiellement collective. Certains colloques ou congrès visent ainsi explicitement à remettre sur le métier, à travailler les conceptualisations de la pratique, pour qu'elles soient le plus appropriées possibles (Martin, \& Baradat, 2003). Les conceptualisations d'une époque sont discutées, affinées, voire modifiées ou contredites, dans un cadre public, et leurs évolutions font l'objet de publications. On peut noter par exemple des évolutions considérables sur le statut de la participation des travailleurs dans l'intervention (Pilnière, \& Lhospital, 1998).

Loin d'une démarche toute faite qu'il s'agirait d'appliquer, les modèles de l'activité de l'ergonome visent ainsi à permettre la construction d'invariants organisateurs d'une activité extrêmement variée dans des environnements différents. La question ne nous semble donc pas être de savoir si les modèles existant aujourd'hui représentent fidèlement la pratique (certainement pas), mais dans quelle mesure ils en soutiennent utilement la construction, ou au contraire ils en entravent le développement. L'enjeu en ergonomie n'est pas de perfectionner les modèles pour qu'ils soient plus fidèles, mais pour qu'ils soient plus opérants (Wisner, 1972).

3. Une question difficile est celle de savoir s'il s'agit seulement d'invariants des récits, ou s'il s'agit vraiment d'invariants des interventions elles-mêmes. Béguin et Clot (2004) le disent à propos des « plans » de Suchman : « le plan est soit une ressource (quand il est produit avant l'action), et il joue alors un rôle d'orientation de l'action, [...] soit un produit a posteriori, une reconstruction sui generis qui vise à prendre l'action pour objet de réflexion après son effectuation ». Ce double visage vaut pour les invariants de l'intervention ergonomique. L'analyse des raisonnements en cours d'intervention serait nécessaire pour trancher cette question. 


\section{2.- Modèles de l'opérateur-ergonome}

Nous avons, ci-dessus, montré l'importance que l'ergonomie est amenée à accorder aux dimensions subjectives dans la modélisation de l'activité de certaines catégories d'opérateurs, en particulier, mais pas seulement, dans les activités de service. Or, force est de constater que la plupart des modèles de l'intervention ergonomique font de l'ergonome un être socio-cognitif, en ce sens que les dimensions physiques d'une part, et subjectives d'autre part de son activité sont peu évoquées.

Des conceptualisations comme celles de l'" analyse de la demande », du « diagnostic », de la « conduite de projet » définissent pour chaque composante de l'intervention des buts, une organisation de la recherche d'information, des règles d'action et de coopération, des outils mobilisables, etc. Elles sont au service des constructions cognitives de l'ergonome, de ses prises de décision quant aux opérations à effectuer dans l'intervention.

De plus, dans les modélisations habituelles en «ergonomie de l'activité », le contexte de l'action pris en compte est, à un degré plus ou moins précis, un contexte social, multiacteur, multilogique, en référence implicite à des descriptions sociologiques plus ou moins riches. Il est admis (dans cette communauté) que la cognition de l'ergonome est mise en œuvre dans un contexte social.

Mais les relations intersubjectives de l'ergonome avec d'autres acteurs, les délibérations auxquelles il/elle est confronté-e, les rationalités qu'il/elle met en œuvre dans l'action, les coûts que cela entraîne pour lui/elle, font l'objet de peu de formalisations.

Il serait possible de paraphraser la phrase de Rabardel citée plus haut : les travaux sur les dimensions cognitives de l'activité de l'ergonome sont si majoritaires qu'ils pourraient finir par donner une représentation distordue de la pratique professionnelle. N'y a-t-il pas là un déficit qui gagnerait à être corrigé, si l'on veut au mieux préparer les futurs ergonomes à leur métier ?

Car d'autres professions en ont fait le constat. Les difficultés d'enseignants du secondaire, de travailleurs sociaux, de médecins généralistes (Davezies, \& Daniellou, 2004) sont parfois sérieuses, avec des risques importants pour leur santé. Elles sont notamment liées à des cursus de formation orientés vers le développement de leur activité cognitive, mais qui les outillent insuffisamment pour les relations intersubjectives et les débats de normes auxquels la pratique les confronte. Les générations anciennes d'ergonomes étaient majoritairement issues de reconversions professionnelles de médecins, psychologues, militants, cadres, qui avaient eu l'occasion dans leur carrière antérieure de construire des compétences dans ces domaines. Les générations actuelles se qualifient en ergonomie principalement en formation initiale. Il est de la responsabilité de la profession, et des lieux de formation, de les préparer au mieux aux expériences que leur métier va les amener à vivre.

\section{4.- L'intervention entre expérimentation et expérience}

Schwartz (1994 [2000]) le rappelait au congrès de la SELF. Toute situation de travail a la double dimension d'une expérimentation réglée et d'une expérience vécue : " toute activité industrieuse est toujours à quelque degré, d'une part analogue à une expérimentation, d'autre part expérience ou "rencontre" » :

- Expérimentation réglée car la situation de travail a été préalablement conçue, planifiée, beaucoup de ses dimensions ont été anticipées, ont fait l'objet de protocoles plus ou moins précis. « Même si c'est un idéal, travailler sur et avec des concepts exige cette standardisation des protocoles » (ibid. p. 540);

- Mais aussi expérience vécue car les anticipations qui en ont été faites n'épuisent jamais les variabilités auxquelles va se trouver confrontée l'activité. Ce sont des personnes particulières, des collectifs spécifiques, avec leur histoire propre, qui vont non pas réaliser, mais accomplir 
(Hubault, 1996) ce qu'il y a à faire. « Dire que l'activité industrieuse est toujours rencontre, c'est rappeler qu'il est impensable, qu'il est absurde d'imaginer neutraliser toutes les variables singulières » (Schwartz, op. cit. p. 541).

Schwartz s'exprimait dans un congrès sur « ergonomie et ingénierie », pour rappeler que ce qui est évidemment vrai pour les concepteurs l'est aussi pour tout opérateur : reconfigurer, resingulariser le milieu d'activité est comme « une vocation de l'humain » (p. 543). Il plaidait pour l'organisation de la « rencontre », à toutes les étapes de la conception, entre ceux qui sont porteurs de l'abstraction, des concepts généralisateurs, et ceux qui sont porteurs des micro-gestions de la variabilité quotidienne.

Nous défendons l'idée que l'activité de l'ergonome en intervention peut faire l'objet du même raisonnement. Pour partie, l'intervention est une expérimentation construite par l'ergonome avec les autres acteurs. Les connaissances, les modèles, permettent d'anticiper certaines des dimensions de cette expérimentation, par exemple des précautions à prendre dans la rédaction du contrat, le type d'association des représentants du personnel, la structuration des relations entre maitrise d'ouvrage et maîtrise d'œuvre, ... Fort heureusement, l'ergonome ne part pas de rien, et les formalisations antérieures permettent d'anticiper certains buts intermédiaires, d'orienter certaines prises d'information (de mettre en place les bons capteurs), de prévoir certains résultats.

Mais, bien entendu, le déroulement réel de l'intervention est loin d'être réductible à la mise en place d'un protocole. L'ergonome particulier qui est là va déployer une activité personnelle qui déborde très largement la mise en place d'une méthodologie définie par avance. Il/elle va être confronté-e à toutes les singularités de la situation, des personnes, collectifs, processus techniques qui la composent, des éléments de contexte. Ce faisant, il/elle se mobilise en tant que personne, (qui a aussi une vie privée), a des relations intersubjectives avec ses interlocuteurs, et est confronté-e à des délibérations parfois difficiles, entre des enjeux contradictoires. L'intervention est aussi expérience vécue.

Les dimensions subjectives de l'activité d'intervention ne sont bien sûr pas niées par les auteurs qui modélisent l'activité de l'ergonome. Mais elles sont peu thématisées, que ce soit par la discipline ou par la profession, comme si elles consistaient en une alchimie privée qui ne relèverait pas d'une formalisation ni d'une mise en débat.

Pourtant, si l'on suit Cerf et Falzon (2005 p. 15), qui proposent de considérer qu'une caractérisation possible de l'activité de l'ergonome est de dire qu'il s'agit d'une activité de service ${ }^{4}$, le moins est de mobiliser pour la décrire des modèles aussi riches que ceux qui sont couramment mis en œuvre pour comprendre le travail dans les services.

\section{5.- L'ergonome-sujet et les débats de normes}

Les modèles de l'activité de l'ergonome, qui ont vocation à être «enseignables » (au sens de Le Moigne, 1995), nous sembleraient devoir prendre en compte au moins trois aspects complémentaires :

1. Le tissu social de l'entreprise n'est pas fait d'interlocuteurs «typiques » (Schutz, 1987) : les chefs d'établissement, représentants du personnel, concepteurs, médecins du travail, etc., que l'ergonome rencontre dans l'intervention, ont certes des fonctions définies par des cadres et des règles que l'ergonome doit connaître. Mais ce sont aussi des personnes particulières, qui ne peuvent pas être ramenées aux seules caractéristiques de leur fonction. Leur intérêt pour les questions de travail, de santé, et les marges de manœuvre qu'ils cherchent à se donner dans ces

4. Nous faisons également référence à la thèse à paraître de Léonard Querelle sur ce thème. 
domaines sont liés à leur histoire personnelle, familiale, sociale, et aux valeurs qui en résultent, tout autant qu'aux responsabilités qui leur sont confiées à un moment donné. Ils sont tous placés dans des débats de normes fortement personnalisés.

2. Si, évidemment, il n'est pas du rôle de l'ergonome de reconstruire ces biographies, l'issue de l'intervention dépendra largement de la mise en résonance entre les actions qu'il/elle proposera, et les préoccupations professionnelles personnalisées de ses interlocuteurs. De ce fait, la méthodologie d'intervention ergonomique ne peut reposer seulement sur des actes publics de l'ergonome (lors des observations, dans des groupes de travail, de pilotage, ...). Nous avons nousmême, dans les années 1985, contribué à diffuser une formalisation de l'intervention où l'accent portait trop exclusivement sur la mise en œuvre de différents types de groupes, soigneusement équilibrés du point de vue de la représentation des acteurs sociaux. C'est un des mérites de Christol (voir par exemple Escouteloup, 2004) d'avoir insisté sur la dimension des «colloques singuliers », entre l'ergonome et ses interlocuteurs pris individuellement, comme composante essentielle de l'intervention. Une vision des relations sociales dans l'entreprise qui en exclurait toute dimension intersubjective serait aussi peu pertinente pour l'ergonome qu'elle l'est pour décrire les processus de négociation entre direction et représentants du personnel (Dugué, 2005).

3. L'ergonome est, comme tout travailleur, soumis à des débats de normes. Il/elle l'est probablement à un degré élevé, dans la mesure même où son action le place au cœur des tensions entre santé et formes d'efficacité, à l'articulation entre unicité de la conception et variabilités du travail quotidien, en contact avec de très nombreux acteurs porteurs chacun de dimensions contradictoires de la performance de l'entreprise, et situant leurs actions dans des "mondes » différents (Béguin, 2005). La survie économique du site est un enjeu aussi respectable que la prévention des troubles musculosquelettiques, et l'ergonome ne peut se préoccuper de l'une sans s'intéresser à l'autre.

La vigueur des débats de normes auxquels l'ergonome est confronté-e dépend certainement des classes de situations dans lesquelles il/elle intervient. La conception de systèmes automatisés, où la productivité n'est pas proportionnelle aux opérations réalisées par les humains, permet parfois de concilier de façon relativement harmonieuse des enjeux de santé et d'efficacité. Les interventions relatives au travail à la chaîne, aux troubles musculosquelettiques, aux centres d'appels, projettent souvent l'ergonome au cœur de tensions beaucoup plus vives.

Les débats de normes, entre « usage de soi par soi » et « usage de soi par les autres », dont l'ergonome est acteur, ne sont d'ailleurs pas que le reflet de ceux qui existent dans l'entreprise. Il/elle a aussi une histoire, une famille, une vie personnelle, qui sollicitent son énergie et sa disponibilité. Il/ elle intervient dans un cadre professionnel qui comporte des contraintes économiques, et qui impose une lisibilité de son action vis-à-vis de ses collègues.

Cette mobilisation subjective de l'ergonome peut évidemment être gratifiante, et contribuer à sa propre santé. Mais cette issue favorable des débats de normes n'est pas donnée d'avance, et l'ergonome peut aussi courir des risques personnels dans le cas contraire.

C'est la raison pour laquelle ces relations intersubjectives et ces délibérations sont l'une des composantes de la pratique professionnelle, pour lesquelles des conceptualisations pertinentes, voire des mises en situation appropriées, dès le stade de la formation initiale, peuvent aider à une construction plus rapide et moins coûteuse des pratiques de chacun.

\section{6.- Domaines de recherche et d'action}

Ces propositions, si on veut bien les suivre, ouvrent sur des champs de recherche, des pratiques d'enseignement, et des débats professionnels. 


\section{1.- La recherche sur les dimensions subjectives dans l'intervention}

L'évolution des modèles généraux de l'activité mis en œuvre en ergonomie, dans le sens d'une meilleure prise en compte des dimensions subjectives, ouvre la voie à un enrichissement des modèles de l'activité de l'ergonome.

Il est possible de mieux décrire quelques-unes des dimensions subjectives en jeu dans l'intervention, en référence notamment à des travaux menés à propos d'autres professions. Nous avions souligné, par exemple, (Daniellou, 1992, pp. 116 sqq), l'intérêt à ce sujet des travaux de Balint (1957) sur les médecins généralistes, et de Devereux (1967) sur les ethnologues. De nombreuses autres études plus récentes sur d'autres professions pourraient certainement être mises à profit.

Comme le médecin, l'ergonome entend une demande qui manifeste le besoin de recourir à un tiers. Cette demande s'accompagne d'une « offre de représentation » du problème, qu'il/elle ne peut pas prendre au pied de la lettre. Il/elle va offrir des moyens de construire le problème en commun avec le demandeur. Cette interaction est susceptible d'entrer en résonance avec de nombreuses dimensions de la biographie de l'ergonome, pour le meilleur et pour le pire.

Comme l'ethnologue, l'ergonome doit identifier le rôle qu'on lui fait jouer, et considérer que ce qu'il/elle observe n'est pas indépendant de sa présence. La culture et les intérêts de l'ergonome vont l'amener à privilégier certains aspects de la situation sur laquelle il/elle intervient. Ce qu'il/elle constate dans l'entreprise peut être fortement anxiogène, et provoquer le déploiement de défenses psychiques, qui affectent la conduite de l'action.

De tels phénomènes pourraient certainement être analysés dans la pratique ergonomique. Cela nécessiterait que l'analyste, par exemple le chercheur qui tente de modéliser la pratique, s'attache particulièrement à certaines dimensions de l'activité de l'intervenant : les « colloques singuliers » de l'ergonome avec ses interlocuteurs, les débats au sein de l'équipe d'intervenants (Jackson, 1998), les discussions avec des pairs, voire les plaisanteries échangées entre collègues, ou les formes de bizutage des jeunes intervenants. Cela supposerait aussi que l'analyste, comme il le ferait avec tout autre opérateur, interroge l'ergonome sur les formes de coûts personnels qu'il/elle ressent dans une intervention donnée ou dans la configuration d'ensemble de ses interventions simultanées. Les ressources que l'ergonome perçoit comme disponibles pour l'aider dans les situations difficiles pourraient aussi être décrites.

\section{2.- La préparation aux dimensions intersubjectives de la pratique}

Sans attendre le résultat de telles recherches, et des modélisations plus précises des débats de normes auxquels l'ergonome est confronté-e dans l'intervention, il est d'ores et déjà possible d'introduire dans l'enseignement une conceptualisation de ces dimensions, en mettant à profit des modèles construits dans d'autres cadres.

Dans notre pratique d'enseignant, nous essayons d'y contribuer avec les ingrédients suivants :

- Une familiarisation des ergonomes-en-formation avec les cadres conceptuels des disciplines voisines qui éclairent les dimensions subjectives de toute activité de travail (nous avons cité la psychodynamique, l'ergologie, la clinique de l'activité, il faut bien sûr y ajouter les théories du stress, la psychologie de la santé, etc.).

- Plus généralement, une découverte des travaux qui soulignent les dimensions intersubjectives de la communication. De nombreux ergonomes en formation ont manifesté l'intérêt qu'ils avaient trouvé à lire Watzlawick (par exemple 1986, et plus généralement l'école de Palo Alto), ou Devereux (op. cit.). Les travaux de Mauss sur le don et le contre-don (Godbout, 2000) ${ }^{5}$, qui se révèlent particulièrement utiles pour analyser les difficultés des médecins généralistes (Davezies, \& Daniellou, 2004), ont aussi toute leur pertinence pour la pratique réflexive des ergonomes. 
- Une évocation, dans la formation, des débats auxquels est confronté-e l'ergonome et des formes de coûts que cela entraîne pour lui ou elle ${ }^{6}$. Les repères déontologiques connus, et leurs limites, peuvent être discutés. Des formes de présence des enseignants ou d'ergonomes-seniors peuvent être organisées, pour assister en temps réel les ergonomes-en-formation soumis à des débats difficiles sur le terrain ${ }^{7}$.

- Une complexification de la modélisation des rapports sociaux. La vision d'une entreprise harmonieuse, où régnerait un mystérieux intérêt général, est aussi inappropriée que la description simpliste de l'opposition de deux forces en présence, celle des « salariés » et celle de la « direction ». Les développements théoriques en sociologie, par exemple autour de Jean-Daniel Reynaud (1995, Terssac de, 2003), permettent de fournir une image d'un grand nombre de forces en présence dans toute organisation, et des régulations multiples qui s'y développent. Il faut probablement, pour la formation des ergonomes, ajouter une réflexion sur l'intrication des dimensions subjectives et des dimensions sociales, notamment dans les rapports sociaux d'entreprise.

- Une réflexion sur le statut des règles dans le travail, et plus généralement dans le fonctionnement des collectifs. Il s'agit notamment de préparer les ergonomes à une analyse bienveillante du travail de l'encadrement, et de leur éviter de confondre abusivement l'exercice normal de l'autorité hiérarchique avec une conduite perverse du cadre concerné.

Il ne s'agit là que de quelques exemples, puisque ce qui est en jeu est l'application, à la compréhension de l'activité de l'ergonome, de l'ensemble des travaux en sciences humaines. Rendant hommage à son maître Philippe Malrieu, Curie (2000 pp. 7-8) écrit : « D’emblée est posée l'affirmation que le rôle de la connaissance est de permettre aux hommes de participer à la construction de leur propre humanité. Cette construction est considérée comme un dépassement de divisions à l'intérieur de soi-même, divisions qui résultent de l'adhésion à des modèles dont l'antagonisme est le plus fréquemment masqué. Ce dépassement suppose d'une part l'acquisition d'instruments intellectuels élaborés par les hommes au cours de leur histoire [...], d'autre part l'engagement dans des relations interpersonnelles diversifiées, et enfin la possibilité pour les hommes de mettre en œuvre des pratiques dans lesquelles s'expriment les conquêtes d'autonomie dont ils ont pu faire l'expérience personnelle. Ce qui pour le sujet donne valeur à l'acte, ce n'est pas sa conformité à des besoins issus d'une quelconque nature biologique programmée ou à des modèles sociaux qui, effectivement préexistent à ce sujet, c'est la participation de cet acte au dépassement des contradictions qui existent à l'intérieur et entre ces deux ordres de déterminants. [...]. Le rôle du psychologue, chercheur ou praticien est de fournir une aide à ce travail de soi sur soi en mettant en lumière ce qui le bloque et en suggérant des prises pour agir sur ces obstacles ». Ici aussi, il nous semble facile de relire cette citation en l'appliquant à l'ergonome comme sujet.

\section{7.- Conclusions}

Une meilleure prise en compte des dimensions subjectives de l'activité de l'ergonome nous semble nécessaire dès la formation des futurs intervenants. Elle suppose que ces dimensions aient droit de cité scientifique, c'est-à-dire que les modèles de l'activité de l'ergonome qui ont la dignité de

5. Celui qui donne plus qu'il ne reçoit crée une dette chez le donataire, en fait un obligé, et donc, d'une certaine façon, l'asservit. Mauss a étudié chez les Maoris le phénomène par lequel celui qui reçoit est envahi de l'esprit du donateur. Chez les Romains, le débiteur qui ne pouvait acquitter sa dette devenait esclave de son créancier.

6. Je suis toujours surpris de l'importance qu'a, pour les ergonomes en formation, le fait qu'on leur enseigne que les ergonomes expérimentés font des cauchemars à propos de leurs propres interventions.

7. Jacques Christol (comm. pers.) formalise cette idée, qu'il désigne par «SOS Amitié ergonomie », en insistant sur le besoin d'une écoute immédiate lorsque l'on est pris sur le terrain dans des contradictions insurmontables. 
« référence légitime » les intègrent progressivement. L'évolution des théories de l'activité nous semble préparer cette ouverture.

Il faut d'ailleurs s'attendre à un bénéfice scientifique et professionnel « secondaire » : mieux outillée pour percevoir les dimensions subjectives de sa propre activité et les gérer, l'ergonome sera sans doute mieux armé-e pour comprendre et prendre en compte les débats de normes à l'œuvre chez ses interlocuteurs, et pour développer des interventions [encore] plus pertinentes.

Mais cette ouverture de l'enseignement est indissociable de celle des communautés professionnelles. Une défense, classique dans de nombreuses professions, veut qu' " un bon professionnel n'a pas de problème, ou, s'il en a, s'en débrouille seul ». La mise en collectif des débats de normes auxquels chacun-e est confronté-e ne peut pas se développer sur un tel terreau. La multiplication des lieux d'échanges entre ergonomes, sous forme de débats sur la pratique, régionaux ou par thèmes, peut être une opportunité pour cette prise en compte des dimensions subjectives de l'intervention, et pour la construction de savoirs collectifs permettant de mieux s'y situer. Une telle voie n'est cependant pas tracée d'avance: entre états d'âme privés dont la mise en débat collectif serait obscène, et dimensions insubstituables de la pratique du métier et de son efficacité, le statut professionnel et scientifique des délibérations des ergonomes reste à construire.

\section{BIBLIOGRAPHIE}

APST (1992). L'évaluation économique à l'épreuve des services: l'activité entre efficacité et efficience. Rapport de recherche pour le Ministère de la recherche et de la technologie, Aix-en-Provence: Université de Provence.

Balint, M. (1957 [1988]). Le médecin, son malade et la maladie. Paris: Payot.

Béguin, P. (2005). Concevoir pour les genèses professionnelles. In P. Rabardel, \& P. Pastré (Eds), Modèles du sujet pour la conception (pp. 31-52). Toulouse: Octarès Editions.

Béguin, P. \& Clot, Y. (2004). L'action située dans le développement de l'activité. @ctivités, 1 (2), 35-49. http:/ /www.activites.org.

Bruner, J.,(1990 [1991]). ...car la culture donne forme à l'esprit. Paris: Eshel.

Canguilhem, G. (1966). Le normal et le pathologique. Paris: PUF.

Cerf, M., \& Falzon, P. (2005). Situations de service : travailler dans l'interaction. Paris: PUF, collection Le Travail Humain.

Clot, Y. (1999). Le travail sans l'homme? Pour une psychologie des milieux de travail et de vie. Paris: La Découverte.

Curie, J. (2000). Travail, personnalisation, changements sociaux. Toulouse: Octarès Editions

Daniellou, F. (1985). Ergonomie et projets industriels. Cours polycopié, Paris: CNAM Laboratoire d'Ergonomie.

Daniellou, F. (1992). Le statut de la pratique et des connaissances dans l'intervention ergonomique de conception. Thèse d'Habilitation à Diriger des recherches. Éditions du Laboratoire d'Ergonomie des Systèmes Complexe, Bordeaux: Université Victor Segalen Bordeaux2.

Daniellou, F. (1999). The ergonomist is a worker? That is the (epistemological) question. In N. Marmaras (Ed.), Strengths and weaknesses, threats and opportunities of ergonomics in front of 2000 (pp. 21-28). Athens: The Hellenic ergonomics society.

Daniellou, F. (1996). Questions épistémologiques soulevées par l'ergonomie de conception. In F. Daniellou (Ed.), L'ergonomie en quête de ses principes (pp. 183-200). Toulouse: Octarès Éditions.

Daniellou, F. (2005). The French-speaking ergonomists' approach to work activity: cross influences of field intervention and conceptual models. Theoretical Issues in Ergonomics Science, 6 (5), 409-427.

Daniellou, F., \& Garrigou, A. (1992). Human Factors in design: sociotechnics or ergonomics. In M. Helander, \& M. Nagamachi (Eds.), Design for manufacturability (pp. 55-63). Londres: Taylor and Francis. 
Davezies, Ph., \& Daniellou, F. (2004). L'épuisement professionnel des médecins généralistes, une étude compréhensive en Poitou-Charentes. Poitiers: Union Régionale des Médecins Libéraux. http://www.urmlpc/Client/Publications_urml.asp

Dejours, C. (1981[1993]). Travail, usure mentale. Paris: Bayard.

Dejours, C. (1995). Le Facteur Humain. Paris: PUF, collection Que sais-je ?

Devereux, G. (1967[1980]). De l'angoisse à la méthode dans les sciences du comportement. Paris: Flammarion, collection Nouvelle Bibliothèque Scientifique.

Dugué, B. (2005). Le travail de négociation. Toulouse: Octarès Éditions.

Escouteloup, J. (Ed.) (2004). Trajectoires d'ergonomes : Jacques Christol, Antoine Laville, Yvon Quéinnec. Bordeaux: Laboratoire d'Ergonomie des Systèmes Complexes, Université Victor Segalen Bordeaux 2

Falzon, P. (1997). La construction des connaissances en ergonomie : éléments d'épistémologie. In Actes du XXXII Congrès de la SELF (pp. 641-654). Lyon.

Godbout, J.T. (2000). Le don, la dette et l'identité. Paris: La Découverte/M.A.U.S.S.

Guérin, F., Laville, A., Daniellou, F., Duraffourg, J., \& Kerguelen, A. (1991, 1997). Comprendre le travail pour le transformer. Lyon: Éditions ANACT.

Hubault, F. (1996). De quoi l'ergonomie peut-elle faire l'analyse ? In F. Daniellou (Ed.), L'ergonomie en quête de ses principes (pp. 103-140). Toulouse: Octarès Éditions.

Jackson, J.M. (1998). Entre situations de gestion et situations de délibération : l'action de l'ergonome dans les projets industriels. Thèse de Doctorat. Éditions du Laboratoire d'Ergonomie des Systèmes Complexes, Bordeaux: Université Victor Segalen Bordeaux2.

Lamonde F. (2000). L'intervention ergonomique, un regard sur la pratique professionnelle. Toulouse: Octarès Éditions.

Le Moigne, J.L. (1995). Les épistémologies constructivistes. Paris: PUF, collection Que sais-je ?

Leplat, J., \& Cuny, X. (1977). Introduction à la psychologie du travail. Paris: PUF.

Martin, C. (2000). Maîtrise d'ouvrage, maîtrise d'œuvre, construire un vrai dialogue: la contribution de l'ergonome à la conduite de projets architectural. Toulouse: Octarès Éditions.

Martin C., \& Baradat D. (2003). Des pratiques en réflexion. Toulouse: Octarès Éditions.

Pastré, P. (2005a). Genèse et identité. In P. Rabardel, \& P. Pastré (Eds.), Modèles du sujet pour la conception (pp. 231-260). Toulouse: Octarès Éditions.

Pastré, P. (2005b). La conception de situations didactiques à la lumière de la théorie de la conceptualisation dans l'action. In P. Rabardel, \& P. Pastré (Eds.), Modèles du sujet pour la conception (pp. 231-260). Toulouse: Octarès Éditions.

Petit, J. (2005). Organiser la continuité du service : intervention sur l'organisation d'une mutuelle de santé. Thèse de doctorat d'ergonomie, Bordeaux: Université Victor Segalen Bordeaux 2.

Petit, J., Querelle, L., \& Daniellou, F. (à paraître). Quelles données pour la recherche sur la pratique de l'ergonome? Le Travail Humain (à paraître).

Pilnière, V., \& Lhospital, O. (Eds.) (1998). Participation, représentation, décisions dans l'intervention ergonomique. In Actes des Journées de Bordeaux sur la pratique de l'ergonomie. Bordeaux: Université Victor Segalen Bordeaux 2.

Pinsky L. (1992). Concevoir pour l'action et la communication : essai d'ergonomie cognitive. Berne: Peter Lang.

Rabardel, P. (1995). Les hommes et les technologies. Paris: Armand Colin.

Rabardel, P. (2005). Instrument subjectif et développement du pouvoir d'agir. In P. Rabardel, \& P. Pastré (Eds.), Modèles du sujet pour la conception (pp. 11-30). Toulouse: Octarès Editions.

Rabardel, P., \& Pastré, P. (Eds.) (2005). Modèles du sujet pour la conception. Toulouse: Octarès Editions.

Reynaud, J.D. (1995). Le conflit, la négociation et la règle. Toulouse: Octarès Éditions.

Schutz, A. (1987). Le chercheur et le quotidien. Paris: Méridiens Klincksieck. 
Schwartz, Y. (1994[2000]). Conférence inaugurale du 29ème congrès de la SELF. In Y. Schwartz (Ed.), Le paradigme ergologique ou un métier de philosophe (pp. 537-548). Toulouse: Octarès Éditions.

Schwartz, Y. (2000). Le paradigme ergologique ou un métier de philosophe. Toulouse: Octarès Éditions.

Terssac, G. de (Ed.) (2003). La théorie de la régulation sociale de Jean-Daniel Reynaud. Paris: La Découverte.

Vergnaud, G. (1996). Au fond de l'action, la conceptualisation. In J.M. Barbier (Ed.), Savoirs théoriques et savoirs d'action (pp. 275-292). Paris: PUF.

Watzlawick, P. (1986[1988]). Comment réussir à échouer. Paris: Seuil.

Wisner, A. (1971[1995]). A quel homme le travail doit-il être adapté ? In A. Wisner (1995), Réflexions sur l'ergonomie (1962-1995) (pp. 47-56). Toulouse: Octarès Éditions.

Wisner, A. (1972[1995]). Le diagnostic en ergonomie ou le choix des modèles opérants, pp 79-102, In A. Wisner (1995). Réflexions sur l'ergonomie (1962-1995) (pp. 79-102). Toulouse: Octarès Éditions.

Wisner, A. (1979). Analyse de la situation de travail, méthodes et techniques. Cours B3, Paris: Laboratoire d'Ergonomie du CNAM.

\title{
RÉFÉRENCEMENT
}

Daniellou, F. (2006). Entre expérimentation réglée et expérience vécue : Les dimensions subjectives de l'activité de l'ergonome en intervention. @ctivités 3 (1), 5-18, http://www.activites.org/v3n1/daniellou.pdf

\begin{abstract}
RÉSUMÉ
Les modèles de l'activité des opérateurs, produits en ergonomie, intègrent de plus en plus une dimension subjective. Les modélisations de l'activité des ergonomes eux-mêmes n'ont pas suivi la même évolution. Pour faciliter la construction et le développement des pratiques professionnelles, il semble nécessaire de modéliser et de faire référence dans les enseignements aux dimensions subjectives de l'activité de l'ergonome.
\end{abstract}

\section{MOTS CLÉ}

Analyse de l'activité, pratique ergonomique, intervention, subjectivité.

\begin{abstract}
RESUMEN
Los modelos de la actividad de los operadores producidos por la ergonomía integran cada vez más a la dimensión subjetiva. Pero, las modelizaciones de la actividad de los mismos ergónomos no han seguido la misma evolución. Para facilitar la construcción y el desarrollo de las prácticas profesionales, resulta necesario modelizar y hacer referencia en la enseñanza, a las dimensiones subjetivas de la actividad del ergónomo.
\end{abstract}

PaLABRAs Clave

Análisis de la actividad, práctica ergonómica, intervención, subjetividad.

Article reçu en décembre 2005, accepté en février 2006. 